\title{
Liderazgo pedagógico, liderazgo docente y su papel en la mejora de la escuela: una aproximación teórica
}

\section{Pedagogical Leadership, Teaching Leadership and their Role in School Improvement: A Theoretical Approach}

\section{Trilce S. Contreras}

Universidad Pedagógica de Heidelberg, Heidelberg, Alemania.

Educadora y Magíster en Liderazgo Educativo.

Recibido: 13-06-16

Aprobado: 08-09-16

\section{Correspondencia}

Email: contrerasmendez.trilce@gmail.com
Citar como:

Contreras, T. (2016). Liderazgo pedagógico, liderazgo docente y su papel en la mejora de la escuela: una aproximación teórica. Propósitos y Representaciones, 4(2), 231-284. Doi: http://dx.doi.org/10.20511/ pyr2016.v4n2.122

(C) Universidad San Ignacio de Loyola, Vicerrectorado de Investigación y Desarrollo, 2016. (c) BY.NC.ND Este artículo se distribuye bajo licencia CC BY-NC-ND 4.0 Internacional (http://creativecommons.org/licenses/by-nc-nd/4.0/). 


\section{Resumen}

Las demandas educativas del siglo XXI hacen indispensable replantearse los modelos tradicionales de gestión y liderazgo en las escuelas y apostar por un enfoque centrado en lo pedagógico, el liderazgo distribuido, el desarrollo participativo de la escuela y el crecimiento profesional docente. El liderazgo pedagógico engloba estos aspectos y se perfila en la investigación educativa como el modelo más efectivo para lograr mejoras sostenidas en la escuela. El liderazgo docente es un elemento esencial de este modelo y se ha acreditado también en los últimos tiempos como rasgo fundamental de la escuela exitosa. Se hace un abordaje teórico-conceptual del liderazgo pedagógico y del liderazgo docente, refiriendo los marcos en los que pueden desarrollarse y su papel en la mejora de la escuela. Se concluye que el profesorado, su potencial de liderazgo y el liderazgo pedagógico conforman una nueva noción integral de escuela que tiene como máxima servir al alumno y su aprendizaje, al desarrollo humano y al desarrollo profesional. Para la transformación de la educación y el desarrollo de la escuela es esencial que profesores y directores sean debidamente capacitados y empoderados, y que tomen conciencia de su rol protagónico en dichos procesos.

Palabras clave: Liderazgo pedagógico, liderazgo docente, mejora escolar, desarrollo escolar, cambio educativo.

\section{Summary}

Educational demands of the 21st century make it indispensable to reevaluate the traditional models of management and leadership in schools and focus on pedagogical aspects, distributed leadership, participative school development and teachers' professional growth. Pedagogical leadership includes these aspects and it is emerging, within the educational research field, as the most effective model to achieve sustained improvements in the schools. Teaching leadership is an essential element of this model and it has also been accredited, in recent years, as a fundamental characteristic of a 
successful school. A theoretical-conceptual approach of pedagogical and teaching leadership is made, presenting the conditions in which they can be developed, and their role in school improvement. Conclusions are the following: Teachers, their leadership potential and pedagogical leadership, define a new comprehensive notion of school which is aimed to serve the students and their learning process, and to promote human and professional development. In order to achieve an educational change and school development, it is essential that teachers and principals are appropriately trained and empowered, and become aware of their key role within such processes.

Keywords: Pedagogical leadership, teaching leadership, school improvement, school development, educational change. 


\section{Introducción}

Educación y transformación son términos omnipresentes en los discursos de sociedades emergentes; innovación y reflexión, palabras clave en las dinámicas de las sociedades modernas. Estas se ven expuestas a cambios constantes, que traen consigo el reto de optimizar de manera permanente estados, procesos y servicios. Las competencias intelectuales, sociales $\mathrm{y}$ emocionales de los individuos son fundamentales para lograr esto y adaptarse al acelerado ritmo de exigencias que se da hoy en día. Ellas son, en esencia, el pilar del éxito o fracaso de una sociedad. Así, atender la demanda educativa de manera satisfactoria y, con ello, el desarrollo continuo del sector educación y de la escuela son asuntos nucleares en todo discurso y práctica de desarrollo. Bastante es sabido que el éxito educativo de una sociedad se traduce en igualdad de oportunidades, acceso al bienestar, integración y desarrollo sostenido. ¿Pero qué caminos debemos trazar hacia tal éxito y el desarrollo continuo de la educación?

La sociedad del conocimiento ha relegado los paradigmas de aprendizaje tradicionales, memorísticos y deterministas, siendo hoy en día demandadas competencias reflexivas, analíticas e interpretativas, que se basan en el razonamiento crítico-constructivo y generan y aprovechan espacios de innovación y optimización, así como formas de trabajo cooperativas y participativas. El desarrollo sostenido parte de y requiere la modernización y transformación de las estructuras y formas de trabajo en el sector educativo, acordes a las demandas de la sociedad que lo aloja, sostiene y genera. Este es el gran reto para la educación y la política de las sociedades emergentes. El mundo es hoy en día, como se afirma en el informe McKinsey, "indiferente a la tradición y a la reputación pasada [...] No perdona las flaquezas e ignora las costumbres o prácticas. El éxito será para las personas y los países que sean rápidos para adaptarse, moderen sus quejas y estén abiertos al cambio. La tarea de los gobiernos será garantizar que los países asuman este desafío" (Barber y Mourshed, 2008, p. 6). 
La investigación sobre eficacia educativa demuestra que la calidad docente es el factor de mayor impacto en el aprendizaje. La formación y el desarrollo profesional del cuerpo docente en ejercicio se vuelven con ello componentes claves en el diseño, desarrollo y mejora de la calidad escolar. Por otro lado, la experiencia de sistemas educativos exitosos sitúa al liderazgo escolar como segundo factor de influencia en los aprendizajes. Tales sistemas presentan una cultura de liderazgo organizacional y una administración escolar enfocada y comprometida con el aprendizaje y el alumno, teniendo como fin último objetivos pedagógicos. La evidencia empírica ha demostrado que una dirección escolar con estos rasgos tiene efectos positivos en los aprendizajes, la calidad de enseñanza y el clima organizacional en la comunidad educativa (Barber \& Mourshed, 2008; Bolívar, 2010; Horn \& Marfán, 2010; Leithwood \& Jantzi, 2008; Marzano, McNulty \& Waters, 2005; Pont, Nusche \& Moorman, 2008).

Barber y Mourshed (2008) indican que el liderazgo pedagógico es, aún en mayor medida, crucial cuando se trata de implementar cambios y transformar sistemas. En este sentido, la formación y el rendimiento docente, así como el liderazgo escolar, son puntos esenciales en la agenda educativa de países en desarrollo, que tienen por delante el gran desafío de elevar, acelerada y significativamente, sus niveles educativos. La evidencia con la que se cuenta hoy en día deja en claro que sin docentes adecuadamente capacitados y de alto desempeño, y sin líderes pedagógicos en la dirección de las escuelas, con la autonomía necesaria para poder decidir y ejecutar medidas en la misma, la buena escuela y el desarrollo escolar tienen pocas posibilidades de realizarse.

Acorde con los paradigmas actuales de desarrollo y manejo organizacional, que fomentan formas de trabajo cooperativa y participativa, y dan pie a la innovación y optimización de recursos, estados y procesos, la escuela ha evolucionado a un punto en el que su desarrollo no puede darse más de manera centralista, diseñado por organismos de mayor jerarquía en el aparato estatal. Este debe partir, más bien, de la propia escuela, las 
competencias, el capital humano y el potencial de cambio interno que esta pueda generar.

La idea principal en esta nueva concepción de escuela es fomentar su desarrollo como organización autónoma y empoderada, que aprende e innova con sus propios recursos y es dirigida de manera democrática y participativa. Fend (1986, como se citó en Rolff, 2012a) habla en este sentido de un cambio de paradigma en relación con lo que hoy se entiende por desarrollo escolar, que resulta del paso de una perspectiva de planeamiento escolar centralista al descubrimiento de la escuela como unidad de diseño y creación. En este nuevo paradigma, cada institución educativa se convierte en el motor principal del desarrollo y en la instancia en la que se determina el éxito o fracaso del sistema educativo. El liderazgo pedagógico, acreditado por la investigación empírica como el más efectivo en materia de rendimiento escolar y de sistemas educativos, es expresión de los nuevos paradigmas de educación y escuela. Encontramos en él un perfil de liderazgo más rico, un genuino liderazgo para el aprendizaje, que a través del liderazgo docente y de comunidades profesionales de aprendizaje se extiende y distribuye más allá de la dirección escolar (Bolívar, 2010). En el presente artículo trabajaremos una aproximación teórica a los conceptos de liderazgo pedagógico y liderazgo docente, refiriendo los marcos en los que pueden desarrollarse y su papel en la mejora de la escuela y del sistema educativo.

\section{Liderazgo pedagógico: un abordaje teórico-conceptual.}

El interés por el tema de liderazgo, habilidades y eficacia directiva en las escuelas nace en el marco de la investigación sobre liderazgo en general y sobre efectividad escolar, pero se cristaliza y profundiza en trabajos más autónomos, recién hacia finales del último siglo. Tanto en la investigación sobre liderazgo en general como en la que concierne específicamente al liderazgo educativo se ha probado la eficacia de estilos participativos, basados en principios sistémicos y de interacción dinámica. Estos difieren claramente de las formas de manejo institucional tradicionales, en las que 
tanto fuera como dentro de la escuela se dan estructuras jerárquicas rígidas, disociadas en sus distintos niveles y enfocadas en asuntos de carácter normativo y administrativo. El manejo organizacional tradicional conlleva en las escuelas a:

- Prácticas rutinarias de enseñanza. El enfoque en lo normativo relega a segundo plano los asuntos pedagógicos, la innovación y el desarrollo de las prácticas de enseñanza. Encontramos, inducidos por el sistema, docentes más preocupados en cumplir con el currículum y avanzar lo planeado para la clase, que en el aprendizaje real del alumno que se está dando. Esto se refleja, por ejemplo, en grandes porciones de clase dictadas de manera frontal por el profesor y en pocas fases de participación activa de los alumnos, lo que descuida el fomento de competencias analíticas y reflexivas.

- Poca permeabilidad e intercambio entre las distintas áreas funcionales de la escuela, que mantienen, por ejemplo, a los docentes apartados de tareas de liderazgo y a los directivos del quehacer pedagógico en las aulas, sumidos en asuntos burocráticos y administrativos. Es común encontrar en este escenario a directores que no brindan soporte al trabajo pedagógico de los profesores, ni se involucran en el aprendizaje en las aulas, ni en el día a día de alumnos y docentes, lo cual genera un clima de trabajo frío y de poca compenetración y entendimiento mutuo. Tales directores pueden pasar días o semanas en su oficina frente al ordenador, revisando papeles e interactuando con el personal administrativo, sin darse una vuelta por los pasillos de la escuela en los recreos, reunirse con los profesores para preguntarles cómo les va en el dictado de clases o hacerles una visita espontánea.

- Un clima de subordinación y prácticas directivas autoritarias, que privan a docentes, alumnos y demás miembros de la comunidad educativa de poder de decisión y participación activa en el manejo 
de la escuela. Esto se refleja, por ejemplo, en una modificación del currículum sin consulta previa y/o participación activa del profesorado en el proceso o en el predominio de instrumentos de gestión de carácter normativo (p. ej., reglamentos de trabajo, tablas de remuneración, etc.) sobre aquellos pedagógicamente funcionales (p. ej., estándares de una buena escuela, buenas prácticas de enseñanza o buenas prácticas directivas, herramientas diagnósticas, encuestas a docentes o padres de familia, formatos para brindar reportes y comentarios constructivos sobre un rendimiento, estándares de buenas prácticas comunicativas en la escuela, etc.). El clima de subordinación repercute negativamente en la motivación, satisfacción personal y nivel de compromiso de los miembros de la comunidad educativa para con la escuela y sus fines, y la falta de participación activa de esta en la conducción de la escuela causa pérdidas de potencial en la generación de ideas, formas de mejora e innovación.

- Personal docente con escasa incentivación y escasas posibilidades de desarrollo profesional y personal, debido a la falta de talleres de capacitación (ofrecidos por la propia escuela), de liderazgo docente, llevado a cabo, por ejemplo, en la conducción de reuniones semanales de profesores que conforman una comunidad de aprendizaje e intercambian experiencias en temas pedagógicos, o de acompañamiento y reporte regular sobre el desempeño pedagógico provisto por el director u otros docentes con mayor experiencia. Como se ha visto, el personal docente y su desempeño pedagógico es el primer factor de influencia en el aprendizaje de los alumnos, por lo que este punto tiene un impacto negativo importante en la práctica educativa de la escuela y sus resultados.

- Falta de cultura de evaluación y mejora continua, reflejada en la ausencia de análisis diagnósticos periódicos en diversos aspectos de la vida escolar y la implementación de proyectos de mejora en base 
a ellos. Como la escuela "A", por ejemplo, no evalúa cómo se están sintiendo sus profesores, no se percata, o simplemente desatiende el hecho de que la mayoría de ellos no ve reconocido su esfuerzo y no instaura una práctica regular en la que el director de la escuela visite algunas clases y se reúna periódicamente con ellos para felicitar logros y/o brindar apoyo pedagógico en aspectos que aún requieran mejora. La falta de una cultura de evaluación y de implementación de proyectos de mejora que repercutan positivamente en el aprendizaje de los estudiantes, como el que ha sido descrito, es un freno importante en el desarrollo escolar y en la optimización de resultados de aprendizaje.

En contraposición a las prácticas y situaciones expuestas, el liderazgo escolar tiende, hoy en día, a enfocarse en lo pedagógico y en la mejora continua y a ser conceptualizado como un fenómeno organizacional en el que se recurre a estructuras horizontales y a principios de distribución entre diversos actores y roles para enfrentar dilemas y retos de complejidad creciente. Ogawa y Bossert (1995) y Manz y Sims (1993 como se citó en Leithwood \& Mascall, 2008). Las tendencias del discurso actual sobre desarrollo escolar derivan principalmente de los modelos de liderazgo transformacional y liderazgo distribuido, cuyas características principales son (Del Valle, 2010; Longo, 2008; Seitz \& Capaul, 2007):

Liderazgo distribuido, basado en principios éticos y morales y en una visión organizacional común.

- Empoderamiento de los miembros de la organización a través de la exigencia y el fomento individual de intelectos y talentos, así como el fomento de redes sociales y comunidades de trabajo.

- Una cultura de aprendizaje e innovación.

- Un alto grado de compromiso de todos los miembros para con la propia superación, los procesos de desarrollo y transformación y las metas organizacionales comunes. 
Para Longo (2008), la capacidad de los directivos de transferir, compartir y desarrollar el liderazgo es fundamental para el cambio y la innovación. Seitz y Capaul (2007) definen el liderazgo escolar como un proceso de interacción entre líderes y colaboradores que se da a distintos niveles de acción y no se restringe únicamente a una posición formal de liderazgo. La relación entre el líder y su equipo se basa en una influencia mutua; debe aflorar por voluntad de ambas partes y aspira a lograr cambios significativos. Cada particularidad situativa (que engloba, por ejemplo, características de la institución, de la tarea, de la situación y/o de las personas involucradas) requiere diferentes dinámicas de relación y, en consecuencia, distintas habilidades directivas. La competencia directiva puede definirse entonces como la capacidad del líder o directivo de actuar de manera efectiva considerando cada particularidad situativa en la que se enmarcan sus acciones. En este sentido, una dirección eficaz en el contexto escolar se revela como un fenómeno complejo que no se puede manejar con criterios uniformes o estandarizados, pero que se ubica bajo ciertas coordenadas y principios de acción.

Siendo así, resulta difícil encontrar en los aportes de la investigación sobre liderazgo escolar definiciones y términos uniformes referentes al concepto de liderazgo pedagógico. En relación con el liderazgo escolar efectivo, se habla a veces simplemente de "liderazgo escolar" pero, también, seguido de "liderazgo pedagógico", o se refieren los términos comunes en el mundo anglosajón: "educational leadership" (liderazgo educativo), "learning-centered-leadership" (liderazgo centrado en el aprendizaje) e "instructional leadership" (liderazgo instructivo). A su vez, muchas veces se refiere en el discurso académico con el término "liderazgo pedagógico" al hecho de asumir y ejercer posiciones directivas en el mundo educativo, sin que esto se relacione de manera implícita o explícita con un determinado modelo de dirección escolar o con formas de dirección cuya eficacia se haya probado. En este trabajo se aborda el concepto de liderazgo pedagógico como el modelo de dirección escolar y gestión educativa, cuya eficacia ha sido reiteradamente probada con soporte empírico en las investigaciones de 
efectividad y liderazgo escolar. Se trata entonces, más que de un modelo de dirección escolar entre muchos otros, del modelo al que, según la evidencia con la que se cuenta hoy en día, deben apuntar los esfuerzos en materia de diseño, dirección y desarrollo de escuela y sistemas educativos.

Hubert (2013) indica en su aporte "12 tesis de una buena dirección escolar" que los buenos directores se comprometen con valores pedagógicos. Son buenos pedagogos, creen y confían en su equipo y, como expertos e impulsores del trabajo en equipo, son ejemplo del accionar cooperativo, no solo en lo que concierne al trato de alumnos y profesores entre sí, sino como máxima pedagógica de la dirección escolar y del día a día en la escuela. En estos marcos, la administración escolar se considera un mero instrumento para alcanzar objetivos pedagógicos genuinos. En el contexto escolar, el objetivo de toda medida es fomentar el aprendizaje y desarrollo de los alumnos, y para que esto funcione, apunta, las acciones se centran en el desarrollo del personal y de las sesiones de aprendizaje, como parte del desarrollo profesional del cuerpo docente. Los objetivos educativos son las premisas determinantes, y esto genera una cultura escolar en la que los asuntos administrativos y los elementos estructurales se hallan siempre subordinados a los principios pedagógicos $\mathrm{y}$, por consiguiente, pueden ser redefinidos en función de estos últimos.

Bolívar (2010) entiende por liderazgo pedagógico la capacidad de ejercer influencia sobre otras personas y movilizar mediante ella a la organización hacia el objetivo común de la mejora de los aprendizajes de los alumnos. La influencia ejercida no se basa en el poder o autoridad formal. Además, explica que "un liderazgo para el aprendizaje toma como núcleo de su acción la calidad de enseñanza ofrecida y los resultados de aprendizaje alcanzados por los alumnos" (Bolívar, 2010, p. 14). En estas líneas de acción, resulta esencial que el equipo directivo cree espacios y entornos que faciliten y apoyen el aprendizaje organizacional y el del profesorado (Bolívar, 2010), lo que repercutirá, a fin de cuentas, en mejores posibilidades de aprendizaje para el alumnado. 
Un aspecto central del liderazgo pedagógico es, entonces, el crear condiciones que fomenten el aprendizaje y la mejora escolar. Aquí, el director cumple una función catalítica (Bolívar, 2010) y no absoluta. En el liderazgo pedagógico, el éxito y el liderazgo se comparten y asumen conjuntamente de manera genuina. El liderazgo se debe entender como "una función que puede ser ejercida por cualquier miembro, más que como un conjunto de responsabilidades reservadas a un individuo" (Bolívar, 1997, p. 38). Se deben encontrar modos que faciliten el consenso sin que esto implique impedir el pensamiento crítico, y se debe fijar la atención y los esfuerzos en los roles y redes que surjan en la escuela, de tal manera que el equipo se vea implicado en las dinámicas de trabajo propias de una cultura sostenida de aprendizaje y desarrollo (Bolívar, 1997).

Horn y Marfán (2010) resaltan como aspectos centrales del liderazgo pedagógico la responsabilidad compartida, el compromiso personal de los involucrados para con las metas conjuntas y los cambios anhelados y el rol del directivo en el fomento de tales actitudes. Para estos autores (Horn \& Marfán, 2010, p. 83), “el liderazgo consistiría en la capacidad de plantear principios de modo que sean tomados por otros como objetivos propios, creándose así un sentido compartido que moviliza a la organización en pro de estos principios comunes". Así, "el liderazgo educativo logra convocar a la comunidad escolar en un proyecto común de mejora, que implica que todos los actores hagan suyo el objetivo de que los alumnos aprendan, y logra orientar el alineamiento de los recursos pedagógicos financieros y humanos en pos de aquel objetivo compartido" (Horn \& Marfán, 2010, p. 84).

Ya en 1994 Dubs afirmaba que el liderazgo pedagógico no se basa en el control ni la supervisión, sino que está al servicio del desarrollo del profesorado y de la escuela. Según este autor, el liderazgo pedagógico debe ser visto como un proceso integral intrínsecamente ligado al desarrollo escolar, en el que se genera un común denominador para el desarrollo personal, las necesidades del profesorado y las metas y visiones de la escuela. Para Brägger y Posse (2007), una de las tareas claves en las direcciones escolares efectivas consiste en impulsar al profesorado a desarrollar sus capacidades. 
Los buenos directores logran, además, crear mediante el reconocimiento al buen rendimiento un clima de optimismo pedagógico, voluntad conjunta de acción y absoluta confianza en el éxito. Se interesan por cada persona que trabaja y aprende en su centro educativo, sean estos alumnos, profesores $\mathrm{u}$ otro tipo de personal (Brägger \& Posse, 2007).

Rolff (2012b) también enfatiza la estrecha relación entre el liderazgo pedagógico y el desarrollo escolar, y la idea de que dicho liderazgo no se basa en la imposición o mandato, sino en el ejemplo y el poder de persuasión. El desarrollo escolar y la escuela, como sistema social orgánico, demandan una noción pedagógica de liderazgo y no una técnico-funcional. Para Rolf (2012b.), una de las principales metas educativas consiste en forjar una escuela que aprende, es decir, una escuela que se desarrolla. Los directivos, siendo instancias a cargo del fomento, la concepción y facilitación de los objetivos trazados, juegan, sin lugar a dudas, un rol esencial en el sistema educativo y el desarrollo escolar Sin líderes pedagógicos competentes a cargo de la dirección de las escuelas, los procesos de cambio tienen chances nulas de implementación exitosa.

Según Murillo (2006), desde un inicio se planteó en la investigación sobre liderazgo educativo un modelo de liderazgo en el que se priorice la eficacia, se diagnostiquen problemas educativos, se oriente al docente, se promueva la supervisión, la evaluación y el desarrollo del personal y se atienda el desarrollo del currículum. En los años ochenta se acuñaría el término de "liderazgo instructivo", que tuvo fuerte repercusión en la dirección escolar y marcó el cambio del enfoque burocrático tradicional de gestión de escuelas hacia un liderazgo centrado en la enseñanza y el aprendizaje. En esta nueva propuesta de liderazgo destacan la generación de un clima propicio para el aprendizaje, el apoyo, fomento y desarrollo profesional de los docentes, el fomento del trabajo en equipo y de la participación de la comunidad escolar, así como una cultura de evaluación para la mejora.

Una segunda evolución importante en la investigación sobre dirección escolar se daría al trasladar el foco de atención de lo que se considera o debe 
considerar la buena escuela y buenas prácticas de liderazgo a, más bien, las condiciones y líneas de acción bajo las cuales se puede transformar y mejorar la escuela (Murillo, 2006). En este sentido, las dimensiones del liderazgo transformacional influyen en la noción y práctica del liderazgo pedagógico, perfilándolo y enriqueciéndolo.

En los últimos tiempos, el liderazgo distribuido se ha vuelto "un nuevo marco conceptual para analizar y enfrentar el liderazgo escolar" (Murillo, 2006, p. 19). Este modelo se centra en la responsabilidad y el liderazgo compartido, así como en el compromiso y la participación de todos los miembros en las acciones emprendidas para alcanzar los objetivos trazados. El modelo de liderazgo distribuido supone, más allá de una simple remodelación de tareas, "un cambio en la cultura, que entraña el compromiso y la implicación de todos los miembros de la comunidad escolar en la marcha, el funcionamiento y la gestión de la escuela". El directivo crea un clima de trabajo participativo, abierto, de cooperación, confianza y respeto mutuo. Así, el liderazgo se vuelve un fenómeno colectivo, "asumido por distintas personas según sus competencias y momentos" (Murillo, 2006, p. 19), y la escuela incrementa su capacidad para resolver problemas e innovar. La esencia del liderazgo distribuido va mucho más allá de la repartición o delegación de tareas. Consiste en aprovechar el potencial colectivo de la organización, sacándoles el mejor partido a los conocimientos, destrezas, esfuerzos e ilusiones de la comunidad escolar (Murillo, 2006).

El rol de los directivos se redefine en torno a estos nuevos marcos, dejando funciones meramente administrativas en segundo plano, para darle protagonismo a un agente visionario, de cambio e incentivo, que sabe aprovechar y desarrollar el potencial de su gente, sirviendo a metas comunes. De este modo, "todos aprenden, se desarrollan personal y profesionalmente [...] La dirección tiene como máxima prioridad las personas, no los papeles ni tareas", y la escuela se convierte en "una verdadera organización de aprendizaje" (Murillo, 2006, p. 21). 
Leithwood y Riehl (2009, p. 20) definen el liderazgo pedagógico como "la labor de movilizar e influenciar a otros para articular y lograr las intenciones y metas compartidas de la escuela". Estos autores se refieren también al fomento intelectual y al acompañamiento individual como dimensiones esenciales del liderazgo pedagógico (ibíd.). Los líderes pedagógicos “apoyan a sus equipos mostrando respeto y consideración por sus sentimientos y necesidades personales" (Leithwood \& Riehl, 2009, p. 27). La evidencia empírica ha podido demostrar que esto influye positivamente en el entusiasmo, optimismo y compromiso de los miembros de la comunidad educativa, cristalizándose en mejores rendimientos. McColl-Kennedy \& Anderson (2002, como se citó en Leithwood \& Riehl, 2009). El desarrollo de las personas, así como la capacidad directiva subyacente de influir positivamente en sus talentos, potenciales, competencias, motivaciones, patrones de pensamiento y actitudes, son dimensiones esenciales del liderazgo pedagógico y de la concepción moderna de escuela y desarrollo escolar.

Basándonos en la bibliografía revisada sobre liderazgo educativo, definimos liderazgo pedagógico como una gestión y conducción de instituciones educativas y/o procesos pedagógicos, cuyo eje fundamental de acción es lograr y garantizar un aprendizaje de calidad y el bienestar de todos los estudiantes. Se basa en una cultura ética, participativa y de innovación y mejora continua, y asume un compromiso con el desarrollo y el bienestar de todas las personas. Sus líneas de acción y rasgos esenciales son:

- Liderazgo y responsabilidad compartida.

- Liderazgo concebido como un proceso dinámico y complejo, en el que se ejerce una influencia positiva en capacidades, motivaciones, patrones de pensamiento, talentos y actitudes, no basada en autoridad o posiciones formales de poder, sino en el ejemplo y el poder de persuasión.

- El aprendizaje exitoso y el desarrollo positivo de los alumnos como fin último. 
- Voluntad conjunta de acción y compromiso para con las metas y visiones de la institución educativa.

- Cultura organizacional de aprendizaje, desarrollo y mejora.

- Acompañamiento personalizado, apoyo, desarrollo, exigencia y fomento del profesorado.

- Empoderamiento de los miembros de la comunidad educativa.

- Cultura de trabajo cooperativa y participativa, basada en valores éticos y pedagógicos.

- Clima de trabajo abierto, tolerante, de optimismo, respeto, apoyo y confianza mutua.

- Trato adecuado de variables emocionales y motivacionales en las dinámicas de trabajo.

- Las personas y su desarrollo son siempre el centro de toda acción.

- Los asuntos administrativos y las dimensiones estructurales están subordinados a los objetivos pedagógicos y las máximas educativas.

La capacidad de liderazgo pedagógico consiste en lograr, bajo estas líneas de acción, que el aprendizaje escolar y la escuela como institución se optimicen de manera permanente. Siendo, en este modelo de liderazgo, el propósito de toda medida el aprendizaje exitoso y el desarrollo óptimo de los alumnos y las personas, y no las tareas, reglas o papeles, el eje de cada acción, las siguientes palabras de un director escolar exitoso de Boston son una buena expresión de su esencia: "Ser docente es ayudar a los niños a aprender. Ser director es ayudar a los adultos a aprender. Por eso es difícil... Yo recorro los pasillos, recorro los pasillos y recorro los pasillos... Sólo reviso mi correo cuando todos los demás ya se fueron" (Barber \& Mourshed, 2008, p. 34).

La capacidad interna de mejora de la escuela depende, en gran medida, de directivos competentes que puedan construirla y fomentarla, creando 
las condiciones propicias para su desarrollo (Bolívar, 2010). Siendo así, la autonomía de la escuela es fundamental para el ejercicio del liderazgo pedagógico, la innovación y el desarrollo escolar. Los directivos deben contar con la autonomía necesaria para decidir sobre líneas de acción y medidas que tendrán un impacto directo en su institución, y para evaluar y monitorear lo implementado.

Una escuela autónoma, participativa y democrática, que aprende y se desarrolla, es el eje en torno al cual gira el liderazgo pedagógico y en el cual se apoya la noción actual de escuela exitosa. Como hemos afirmado, en el contexto educativo moderno la escuela es la unidad motriz del desarrollo y de la calidad del sistema educativo y, con ello, la instancia en la que se decide, día a día, el éxito o fracaso del mismo. En este sentido, es indispensable para la innovación y la mejora de la calidad educativa que se empodere y se le den las herramientas necesarias para que pueda desarrollar su capacidad interna de liderazgo. Solo así podrá sacarle el mejor provecho a la autonomía de gestión que se le brinde, y los esfuerzos de todos los actores del sector y de los miembros de la comunidad educativa podrán canalizarse en aprendizajes estudiantiles exitosos. Esto nos lleva a concluir que sin un liderazgo distribuido y participativo por parte de la política educativa, y sin el correspondiente empoderamiento de cada comunidad escolar, ni la escuela autónoma, ni el liderazgo pedagógico, ni el desarrollo de la escuela y de la educación son posibles. La mejora de la escuela solo puede partir de la mejora y el desarrollo de las capacidades de las personas que la conforman.

\section{Liderazgo docente en el marco del liderazgo pedagógico y del desarrollo escolar.}

Como se afirma en el informe McKinsey (Barber \& Mourshed, 2008, p. 15), "la calidad de un sistema educativo se basa en la calidad de sus docentes". El paradigma educativo moderno exige, tanto a docentes como a alumnos e instituciones educativas, que vivan y fomenten una cultura de aprendizaje y mejora, que cuenten con la competencia reflexiva necesaria para ello, que 
estén abiertos a la innovación y que presenten una motivación intrínseca, así como actitudes positivas hacia el desarrollo individual y colectivo. Si el liderazgo escolar debe ser ejercido de manera participativa, si los profesores son actores centrales en el sistema educativo y si se aspira a la mejora del mismo, entonces es menester que ellos se conviertan en agentes activos del cambio y asuman y fomenten, junto con los directivos, el liderazgo pedagógico. El rol de liderazgo en el docente es, según Hutchinson (2005), clave en la reforma educativa. Los conocimientos, competencias, actitudes y motivaciones del profesorado se vuelven con esto importantes anclas e impulsores del desarrollo escolar, siendo de suma relevancia para la calidad educativa que no sean descuidados o desatendidos.

El desarrollo profesional es la base para la mejora de la práctica educativa, y es esencial para el crecimiento, la experiencia y el desarrollo de las habilidades del profesorado (Helterbran, 2010). La implementación de un concepto escolar orientado a lo pedagógico y de entornos centrados en el aprendizaje exige a profesores y directores altos niveles de profesionalismo y compromiso (Istance \& Dumont, 2010). Para Henderson y Barron (1995), el cambio escolar efectivo depende del ejercicio de roles de liderazgo adecuados. La demanda de profesionalidad y habilidades directivas en el docente parte de la necesidad de tener líderes altamente competentes en las escuelas que se involucren donde haya dificultades y tengan la capacidad de enfrentarlas y solucionarlas de manera sostenida y colectiva. Howey (1988, como se citó en Henderson \& Barron, 1995).

Ante este panorama, además del liderazgo pedagógico, concretamente el liderazgo docente ha ido ganando cada vez más atención y protagonismo tanto en la investigación y los discursos como en las prácticas de desarrollo escolar y mejora educativa. La importancia del liderazgo docente radica en el rol fundamental que tiene el profesor en el liderazgo pedagógico de la escuela, su funcionamiento en general y sus procesos centrales: la enseñanza y el aprendizaje (York-Barr \& Duke, 2004). Es también, en esencia, expresión de liderazgo distribuido y formas de trabajo cooperativas. 
El ejercicio de roles de liderazgo por parte de los docentes implica que el liderazgo, la responsabilidad y las visiones en la escuela son compartidas (Nappi, 2014; Kurtz, 2009). A su vez, el docente líder comparte con otros sus recursos, experiencia y expertise, de tal forma que los recursos individuales enriquezcan y refuercen los colectivos (Nappi, 2014). De esta manera se desarrolla el potencial interno de rendimiento de la escuela, piedra angular del desarrollo educativo sostenido.

Pero el concepto de liderazgo docente va más allá del hecho de que los profesores asuman posiciones formales o tareas de liderazgo. Se trata, más bien, de que el docente se vuelva un verdadero y comprometido representante de la cultura de aprendizaje y mejora vivida en la escuela y desarrolle actitudes y prácticas de liderazgo individuales, sin que estas necesariamente respondan a una posición formal en el organigrama de la escuela. Como afirma Nappi (2014), el “docente líder" no es un cargo asignado, sino un rol que el individuo asume por sí mismo. Para Helterbran (2010), el liderazgo docente no reside en esencia en los cargos más formales, sino en que el docente vea las necesidades, identifique problemas y tome las riendas de la solución por sus propios medios. Este escenario tiene pocas chances de darse mientras los profesores no tomen conciencia sobre sus propias capacidades y roles de liderazgo, y mientras no desarrollen las competencias y la confianza que se requieren para el ejercicio de un liderazgo pedagógico efectivo (Helterbran, 2010). Es tarea de los directivos y demás actores en posiciones formales de liderazgo fomentar el liderazgo docente y crear las condiciones necesarias para que este aflore, convirtiéndose en su eje y soporte (Helterbran, 2010).

Los trabajos que abordan el concepto de liderazgo docente sugieren que el profesor líder tiene la capacidad de fomentar e incentivar a las personas; que asume roles de liderazgo dentro y fuera del salón de clases, en el colegio, en beneficio de los alumnos y de otros profesores, tomando decisiones, fomentando la excelencia, el profesionalismo, el desarrollo de los procesos de enseñanza y aprendizaje y de la escuela en general, y aporta al liderazgo escolar de diferentes maneras, se identifica con comunidades de profesores 
líderes y aprendices, aporta activa y significativamente a estas, fomenta el trabajo en equipo, es ejemplo de clase exitosa e influye en otros promoviendo la mejora (York-Barr \& Duke, 2004; Seitz \& Capaul, 2007; Wasley, 1991; Katzenmeyer \& Moller, 2001; Lambert, 1998; Muijs \& Harris, 2003). Le es importante lo que hace, cómo lo hace y de qué manera influye lo que hace en el aprendizaje de sus alumnos (Kurtz, 2009).

Para York-Barr \& Duke (2004), los profesores lideran manteniendo el foco de atención en los procesos de enseñanza y aprendizaje, y estableciendo relaciones constructivas y de confianza. Influyen en el desarrollo de personas, comunidades de trabajo y capacidades organizacionales de tal manera que este se refleje en la mejora de los aprendizajes. Para Ash \& Persall (2000, como se citó en Muijs \& Harrys, 2003), los docentes líderes son profesores expertos que, si bien pasan la mayoría del tiempo en el salón de clases, asumen roles de liderazgo en distintos momentos y de distintas formas, siguiendo los principios del liderazgo pedagógico. Kurtz (2009) resalta la posición clave de los docentes para implementar cambios, ya que pueden "simplemente" entrar a la clase y realizarlos. Ni el Estado ni las administraciones regionales o locales cuentan con la información de primera mano con la que cuentan los profesores (Kurtz, 2009) en lo que a materia de enseñanza-aprendizaje se refiere. Siendo así, los aportes, opiniones y capacidades de los docentes son valiosos y determinantes para el diseño, implementación y monitoreo de procesos de mejora escolar.

Day y Harris (2003, como se citó en Mujis \& Harris, 2003) proponen cuatro dimensiones del liderazgo docente:

- Trasladar los principios de mejora escolar a la práctica educativa en los salones de clase.

- Asegurar el liderazgo participativo, en el que todos los docentes se sienten parte de la mejora y el desarrollo, trabajando con sus colegas y guiándolos hacia las metas colectivas. 
- Cumplir un rol mediador, mediante el cual transfieren los conocimientos, informaciones y experiencia con los que cuentan.

- Establecer relaciones estrechas entre profesores, que posibiliten el aprendizaje mutuo.

El liderazgo pedagógico implica, en esencia, el liderazgo docente, siendo metas de ambos la optimización de los procesos de enseñanza y aprendizaje, el desarrollo escolar participativo y el crecimiento de la escuela como organización que aprende. En el marco del liderazgo pedagógico, el liderazgo docente es una estrategia central para convertir a la escuela en una organización profesional de aprendizaje (Mujis \& Harris, 2003; Katzenmeyer $\&$ Moller, 2001). Así, el liderazgo docente trae aportes esenciales al desarrollo escolar y la mejora de la calidad educativa (Seitz \& Capaul, 2007).

Los efectos positivos del liderazgo docente se muestran, en primera instancia, en el desarrollo profesional de los mismos profesores. Las prácticas y actitudes de liderazgo impulsan de manera significativa su desarrollo al expandir su noción de aprendizaje, enseñanza y escuela y al concientizarlos sobre el hecho de que el ejercer liderazgo y el aprender están intrínsecamente ligados (York-Barr \& Duke, 2004). La evidencia empírica indica que el liderazgo docente tiene un impacto positivo en la autoestima y satisfacción de los profesores, lo que conlleva a incrementar su motivación, tener mejores rendimientos (Mujis \& Harris, 2003) y, con ello, a mejores resultados de aprendizaje de los estudiantes.

Para que el liderazgo docente tenga lugar, se requiere el apoyo activo de los directivos, crear espacios de aprendizaje y poner a disposición tiempo y recursos (York-Barr \& Duke, 2004). Como se ha afirmado, es responsabilidad de los directivos y demás actores en posiciones formales de liderazgo que se creen estas condiciones. Ellos están en la posición más favorable para apoyar y estimular a los profesores, darles credibilidad, reconocimiento y aprecio, generar chances para el liderazgo distribuido y el desarrollo, y crear espacios de intercambio y cooperación (Hutchinson, 2005; Kurtz, 2009). Pero los 
protagonistas del desarrollo del liderazgo docente en la escuela son, en efecto, los mismos profesores. Helterbran (2010) señala que el liderazgo distribuido requiere, tanto de los directores como de los profesores, actitudes y líneas de acción pertinentes. Visión, ganas y apoyo son elementos necesarios para hacer que el liderazgo docente aflore e impulsarlo (Helterbran, 2010). El liderazgo docente no consiste, a fin de cuentas, en poner a disposición de los directores una mano derecha para el cumplimiento de sus obligaciones (Helterbran, 2010), sino que da vida, junto al liderazgo pedagógico, a un concepto integral de escuela en pro del aprendizaje, el desarrollo humano y el desarrollo profesional, que tiene como máxima formas de trabajo cooperativas y participativas.

No habrá milagros ni pociones mágicas para solucionar los problemas de ningún sistema educativo (Helterbran, 2010), pero un liderazgo distribuido y profesores valorados, bien capacitados y comprometidos pueden canalizar los esfuerzos hacia resultados importantes. Si se busca un cambio educativo efectivo, entonces la política debe apostar y comprometerse con estos objetivos.

\section{Conclusiones}

Ante el nuevo panorama educativo y las demandas de la sociedad del siglo XXI es indispensable, para la mejora de la escuela, replantearse los modelos de gestión y liderazgo tradicionales y apostar por un enfoque centrado en lo pedagógico, en el liderazgo distribuido y en el desarrollo docente. La evidencia más reciente sugiere que ese es el camino más efectivo para lograr mejoras sostenidas en la calidad de enseñanza, en la gestión de las escuelas y, con ello, en el aprendizaje de los estudiantes y el sistema educativo en general.

Bajo este nuevo enfoque, el liderazgo y el desarrollo escolar son asuntos que competen a toda la comunidad educativa, y los profesores, como actores centrales de esta, deben involucrarse activamente en ellos y fomentarlos. Esto nos remite al concepto de liderazgo docente, que ha ganado creciente 
importancia en los últimos años y se perfila, hoy en día, como elemento esencial del liderazgo pedagógico y de la escuela exitosa. Recopilando aportes de la literatura sobre liderazgo y desarrollo escolar, se ha realizado un abordaje teórico del concepto de liderazgo pedagógico, resaltando su papel en la mejora de la educación y de la escuela. Como resultado se propuso una definición propia y un listado de características esenciales. Abordando también el concepto de liderazgo docente como elemento fundamental del liderazgo pedagógico, llegamos a la conclusión de que el profesorado, su potencial de liderazgo y el liderazgo pedagógico conforman una nueva noción integral de escuela que tiene como máxima servir al alumno y su aprendizaje, al desarrollo humano y al desarrollo profesional. Esta nueva escuela es la impulsora de su propio desarrollo y motor principal del desarrollo del sistema educativo al que sirve.

Se considera de fundamental importancia que todo profesor y director sea consciente de que el liderazgo pedagógico tiene un enorme potencial, de repercutir positivamente, en el rendimiento del sistema educativo, ya que ellos son actores claves en hacer que dicho potencial sea aprovechado. Que el liderazgo pedagógico y el liderazgo docente se realicen y establezcan en la práctica educativa depende, en gran medida, de decisiones políticas mediante las cuales los diversos actores del sistema educativo sean debidamente capacitados y empoderados. Pero que profesores y directores sean conscientes de su potencial de influencia y su rol central en el cambio educativo, y de las maneras en las que pueden satisfacer las demandas que estos traen consigo, es ya una condición importante y punto de partida para el mismo. Para renovar prácticas educativas tradicionales y contribuir al desarrollo de la escuela del siglo XX se recomienda:

- Concientizar mediante talleres a los diversos actores de la comunidad educativa sobre el concepto y la función de la escuela moderna y la necesidad del cambio participativo. Analizar conjuntamente en estos talleres la situación actual de la escuela y, en base a ella, sentar visiones y objetivos de mejora. 
- Instaurar prácticas directivas de asesoría regular a docentes, de organización de actividades escolares y realización de proyectos de mejora con la participación de diversos actores de la comunidad educativa, así como de conferencias de desarrollo escolar en las que periódicamente se actualicen diagnósticos de la situación de la escuela, se identifiquen logros y se fijen nuevas metas y planes de acción de mejora.

- Formar comunidades de aprendizaje entre los profesores que trabajen temas pedagógicos en reuniones periódicas, prevean asesorías y visitas de clase mutuas, y generen redes de confianza y apoyo.

- Transferir liderazgo a los docentes, demandando su participación activa en la toma de decisiones, requiriendo la dirección de comunidades de aprendizaje, adjudicando roles de mentoría y mediación (entre alumnos, entre padres de familia y el equipo directivo, entre otros profesores, entre alumnos y otros docentes, entre alumnos y el equipo directivo, etc.) y/o creando oportunidades de especialización académica, como por ejemplo: resolución de conflictos, prevención de conductas inadecuadas, gestión educativa, investigación, desarrollo de currículum, etc.

Asimismo, para futuras investigaciones se recomienda generar más evidencias de tipo cualitativo que ayuden a comprender las situaciones particulares de gestión y liderazgo en las escuelas, así como los problemas y desafíos a los que estas se enfrentan; y realizar estudios cuantitativos sobre el impacto que medidas ejecutadas vayan teniendo, de tal forma que estas puedan ser modificadas u optimizadas según las necesidades particulares de cada caso. En vista del rol clave que ejercen los docentes en el liderazgo y la práctica educativa, recomendamos también dirigir esfuerzos de investigación -tanto cualitativa como cuantitativa- en conocer y entender concretamente la situación de estos y su labor en las escuelas: ¿Cuáles de sus fortalezas y debilidades influyen de qué manera en la enseñanza? ¿Qué implicaría esto para las propuestas de formación docente en universidades e institutos? ¿Qué 
opinan los profesores sobre su quehacer diario en las escuelas? ¿Qué les perturba sobremanera o se les hace más difícil? ¿Qué les hace falta? ¿Qué los motiva sobremanera? Preocuparse por un personal docente competente, competitivo, satisfecho, motivado y comprometido es esencial para llevar a la escuela por buen rumbo y desarrollar su capacidad interna de liderazgo y mejora sostenida.

\section{Referencias}

Barber, M., \& Mourshed, M. (2008). Cómo hicieron los sistemas educativos con mejor desempeño en el mundo para alcanzar sus objetivos. Santiago de Chile: Editorial San Marino.

Bolívar, A. (1997). Liderazgo, mejora y centros educativos. En A. Medina (Ed.), El liderazgo en educación (pp. 25-46). Madrid: UNED.

Bolívar, A. (2010). El liderazgo educativo y su papel en la mejora: una revisión actual de sus posibilidades y limitaciones. Psicoperspectivas, 9(2), 9-33. http://dx.doi.org/10.5027/psicoperspectivas-Vol9-Issue2fulltext-112

Brägger, G., \& Posse, N. (2007). Instrumente für die Qualitätsentwicklung und Evaluation in Schulen (IQES) - Handbuch einer guten Schule. Recuperado de https://www.iqesonline.net/index.cfm?id=6a5a582ce0c6-b4e6-23c2-7a8b70ed6115

Del Valle García, I. (2010). Liderazgo distribuido, una visión innovadora de la dirección escolar: una perspectiva teórica. Omnia, 16(3), 19-36.

Dubs, R. (1994). Die Führung einer Schule. Stuttgart: Franz Steiner Verlag.

Helterbran, V. (2010). Teacher leadership: overcoming "I'm just a teacher" syndrome. Education, 131(2), 363-371. Recuperado de https://www. questia.com/library/journal/1G1-251534611/teacher-leadershipovercoming-i-am-just-a-teacher

Henderson, M. \& Barron, B. (1995). Leadership challenges for classroom teachers. Revista Education, 116(1), 62-64. Recuperado de http://search. proquest.com/openview/3f6e610764b213ee0e4c382465c3b9e9/1?pqorigsite $=$ gscholar 
Horn,A., \& Marfán, J.(2010). Relación entre liderazgo educativoy desempeño escolar: revisión de la investigación en Chile. Psicoperspectivas, 9(2), 82-104.

Huber, G. (2013). Zwölf Thesen zur guten Schulleitung. En G. Huber, (Ed.), Jahrbuch Schulleitung 2013. Befunde und Impulse zu den Handlungsfeldern des Schulmanagements (pp. 3-11). Colonia: Wolters Kluwer / Carl Link.

Hutchinson, C. (2005). The Teacher as Leader: Transformational Leadership and the Professional Teacher or Teacher-Librarian. School Libraries in Canada, 24(1), 73-79. Recuperado de http://connection.ebscohost. com/c/articles/16746531/teacher-as-leader-transformational-leadershipprofessional-teacher-teacher-librarian

Istance, D., \& Dumont, H. (2010). Future directions for learning environments in the $21^{\text {st }}$ century. En H. Dumond, D. Istance \& F. Benavides, (Eds.), The Nature of Learning. Using research to inspire practice (pp. 317336). París: OECD.

Katzenmeyer, M., \& Moller, G. (2001). Awakening the Sleeping Giant. Helping Teachers Develop as Leaders. Thousand Oaks, CA: Corwin.

Kurtz, S. (2009). TeacherLeadership.Leadership,39(1), 12-14. Recuperadode http://web.a.ebscohost.com/ehost/pdfviewer/pdfviewer?sid=ae72c2c7fd21-4d55-9b4b-07fde9ca0e99\%40sessionmgr4005\&vid=3\&hid=4201

Lambert, L. (1998). Building Leadership Capacity in Schools. Alexandria, VA: ASCD.

Leithwood, K., \& Jantzi, D. (2008). Linking leadership to student learning: the contribution of leader efficacy. Educational Administration Quarterly, 44(4), 496-528. http://dx.doi.org/10.1177/0013161X08321495

Leithwood., K. \& Riehl, C. (2009). ¿Qué sabemos sobre liderazgo educativo? En K. Leithwood (Ed.) ¿Cómo liderar nuestras escuelas? Aportes desde la investigación (pp. 17-33). Santiago de Chile: Fundación Chile.

Leithwood, K., \& Mascall, B. (2008). Collective Leadership Effects on Student Achievement. Educational Administration Quarterly, 44 (4), 529-561. http://dx.doi.org/10.1177/0013161X08321221 
Longo, F. (2008). Liderazgo Distribuido, un elemento crítico para promover la innovación. Revista Capital Humano, 21(226), 84-91.

Marzano, R., McNulty, B., \& Waters, T. (2005). School leadership that works: From research to results. [Liderazgo escolar efectivo: investigación y resultados]. Alexandria, VA: Association for Supervision and Curriculum Development.

Muijs, D., \& Harris, A. (2003). Teacher Leadership-Improvement through Empowerment? An overview of the literature. Educational Management \& Administration, 31, 437-448. http://dx.doi. org/10.1177/0263211X030314007

Murillo, F. (2006). Una dirección escolar para el cambio: del liderazgo transformacional al liderazgo distribuido. Revista Electrónica Iberoamericana sobre Calidad, Eficacia y Cambio en Educación, 4(4),11-24. Recuperado de http://www.rinace.net/arts/vol4num4e/art2_ htm.htm

Nappi, J. (2014). The teacher leader: improving schools by building social capital through shared leadership. Revista Delta Kappa Gamma Bulletin, 80(4), 29-34. Recuperado de https://www.questia.com/library/ journal/1P3-3399899341/the-teacher-leader-improving-schools-bybuilding

Pont, B., Nusche, D., \& Moorman, H. (2008). Improving school leadership. París: OECD.

Rolff, H. (2012a). Grundlagen der Schulentwicklung. En Buhren, C./Rolff, H. (Ed.), Handbuch Schulentwicklung und Schulentwicklungsberatung (pp. 12-39). Weinheim y Basilea: Beltz.

Rolff, H. (2012b). Die Rolle der Schulleitung in Schulentwicklungsprozessen. En C. Buhren, y H. Rolff (Ed.), Handbuch Schulentwicklung und Schulentwicklungsberatung (pp. 203-221). Weinheim y Basilea: Beltz.

Seitz, H., \& Capaul, R. (2007). Schulführung und Schulentwicklung. Theoretische Grundlagen und Empfehlungen für die Praxis. Berna, Stuttgart y Viena: Haupt.

Wasley, P. (1991). Teachers Who Lead: The Rhetoric of Reform and the Realities of Practice. New York: Teachers College Press. 
York-Barr, J., \& Duke, K. (2004). What Do We Know about Teacher Leadership? Findings from Two Decades of Scholarship. Review of Educational Research, 74(3), 255-316. http://dx.doi. org/10.3102/00346543074003255 